\title{
Perfiles del participante en LMOOC: análisis bibliográfico y estudio de caso
}

\author{
Participant profiles in LMOOCs: a literature review and case study
}

\author{
Paz Díez-Arcón \\ Universidad Nacional de Educación a Distancia
}

\begin{abstract}
Resumen
La enseñanza de lenguas a través de cursos masivos (LMOOC) tiene características distintivas del resto de propuestas formativas en línea. Los cursos que funcionan en la actualidad tienen criterios evaluativos más cercanos a métodos tradicionales que suponen la asociación de éxito con la compleción del curso, lo que desemboca en una preocupación por el propio formato masivo al no reflejar altos niveles de compleción. También ofrecen tareas evaluativas que no promueven la práctica real del idioma. Mediante el estudio de perfiles socio-económicos y de comportamiento en línea se comprueba que los participantes no se involucran en el curso, pero son activos durante el tiempo que permanecen en él. Los resultados implican que es necesaria la adquisición de un conocimiento más amplio del participante que muestre si consigue sus propósitos de aprendizaje y así permitir una posible disociación entre el binomio compleciónéxito. Por otra parte, se sugieren alternativas para un diseño del contenido que prime la práctica de la lengua meta.
\end{abstract}

Palabras clave: Aprendizaje de lenguas; MOOC; Perfiles de comportamiento; Perfiles demográficos; Éxito formativo

\begin{abstract}
Language learning through massive open online courses (LMOOCs) have distinctive features from the rest of online formative proposals. The courses currently working have assessment criteria closer to traditional methodologies, where success is associated with course completion, which leads to a concern about the massive format itself, as high completion levels are not achieved. They also offer assessment tasks not promoting the real practice of the language. Throughout the study of socio-economic and online behavioral profiles it is noticed that participants are not engaged with the course, but they are active during the time they stay on it. The results imply that the acquisition of a broader knowledge about participants that reflects if they achieve their learning goals is needed and, so that, permit a possible dissociation between success and completion. Moreover, alternatives for a content design prioritizing the practice of the target language are suggested.
\end{abstract}

Keywords: Language learning; MOOC; Behaviour profiles; Demographic profiles; Formative success 


\section{INTRODUCCIÓN}

El siguiente estudio realiza un análisis bibliográfico sobre estudios de caso en MOOC (Massive Open Online Course) de lenguas extranjeras en lo que a perfiles de comportamiento y socioeconómicos se refiere. También se estudia el caso del LMOOC (Language Massive Open Online Course) "How to succeed in the English B1 level exam" ( $1^{a}$ edición) para observar los perfiles mencionados en este caso específico y poder contrastar los resultados con la tendencia general. Mediante la triangulación de datos cualitativos y cuantitativos se definen los perfiles, lo que permite responder a dudas ante los modelos pedagógicos actuales y la respuesta a estos por parte de los participantes. A través de los resultados se realizan propuestas concretas para afrontar los retos de acceso, diseño y participación en LMOOC.

\section{OBJETIVOS Y PREGUNTAS DE INVESTIGACIÓN (PI)}

El objetivo general es trazar el perfil del participante LMOOC mediante una revisión de la literatura sobre los perfiles de sus participantes para comprender y afrontar las limitaciones asociadas a este formato. El segundo objetivo surge con el paso de la generalidad a lo concreto mediante el estudio del caso "How to succeed in the English B1 Level exam" (1 ${ }^{a}$ edición). Las preguntas de investigación derivadas son: PI1) ¿Qué características socio-económicas definen de manera mayoritaria al participante LMOOC?; PI2) ¿Cuál es el perfil de aprendiz dominante de los LMOOC teniendo en cuenta patrones de comportamiento?; PI3) ¿Cuáles son los perfiles predominantes en el caso del LMOOC "How to succeed in the English B1 Level Exam" teniendo en cuenta factores socio- económicos y de aprendizaje?, y PI4) ¿Hasta qué punto sigue el caso específico la tendencia generalizada en los MOOC de lenguas establecida en las preguntas de investigación 1 y 2 ?

\section{MARCO TEÓRICO}

\section{Papel y principios básicos de los MOOC: democratización y acceso libre a la enseñanza y el conocimiento}

Los MOOC han sido definidos como gratuitos, masivos y ubicuos (Cormier y Siemens, 2010) y han tomado un papel relevante como evolución natural del movimiento de aprendizaje abierto (Open Learning Movement) (Atenas, 2015). Teniendo en cuenta las características intrínsecas de los MOOC, se ha teorizado con la idea de que han de favorecer la democratización de la educación y, por lo tanto, al derecho universal a esta (Vázquez-Cano et al., 2016), pero existen diversos motivos por los cuales no se cumple este propósito. La publicidad que se le da a los 
cursos masivos no está enfocada en la dirección de la población que más podría beneficiarse de ellos (Bárcena et al., 2014). También existen barreras para acceder a ellos como en el caso de los LMOOC, donde no queda claro cómo los aprendices que no tienen un nivel fluido del idioma pueden involucrarse con el contenido (Türkay et al., 2017). Este hecho supone "...un esfuerzo cognitivo mucho mayor que en otras disciplinas MOOC.” (Martín-Monje, 2019, p. 39). Otro ejemplo es la necesidad de considerar cómo trabajar con un público tan masivo teniendo en cuenta sus diferentes experiencias y creencias educativas (Ross et al., 2014). También en referencia a lo masivo o multitudinario, Knox (2014) explica cómo la idea de percibir al participante en cursos masivos como una única entidad puede provocar que pase desapercibido el hecho de que la población que accede a estos cursos es altamente heterogénea.

\section{Pros y contras de la utilización de los MOOC en educación}

Una de las preocupaciones que surgen sobre los MOOC es su alta tasa de abandono (Martín-Monje, 2019). Según Kizilcec et al. (2013) tan solo un 10\% aproximadamente de los estudiantes que se inscriben logran completarlo. Un mayor conocimiento sobre las características del participante en el MOOC de idiomas mediante recursos tecnológicos que monitorizan su comportamiento en línea (Ozan et al., 2018), tiene que lograr el que por parte de la comunidad docente se deje de percibir la educación online como el reto de educar a completos desconocidos (Castrillo et al., 2018).

\section{MOOC para la enseñanza de lenguas extranjeras o LMOOC: necesidades pedagógicas específicas, características adaptativas y relación con el Enfoque Comunicativo}

Los LMOOC contienen una combinación de elementos de los dos tipos de cursos masivos identificados atendiendo a la pedagogía utilizada en ellos: cMOOC y los xMOOC (Martín-Monje et al., 2018). Los primeros trabajan un enfoque conectivista donde se aprovechan las interacciones entre los diferentes participantes y donde juegan un papel esencial los medios sociales. Por otra parte, los xMOOC tienen un enfoque conductista basado principalmente en la interacción con el material y los contenidos (Conole, 2016)

Los LMOOC tienen la particularidad de no solo estar basados en el conocimiento sobre reglas o aspectos que se adquieren por transferencia pasiva, sino que al tener como fin el acto comunicativo, necesitan del desarrollo de todas las destrezas del idioma y de las habilidades comunicativas del lenguaje, por lo que las 
plataformas tienen que adaptarse a estas necesidades y ofrecer materiales basados en las competencias lingüísticas que se puedan adaptar a las preferencias de los estudiantes (Martín-Monje et al., 2018). Según Kizilcec et al. (2013) las características adaptativas pasan por dar cobertura a participantes de diferentes bagajes, con diversas intenciones o motivaciones de cara al aprendizaje y que puedan encontrar limitaciones personales o técnicas durante la instrucción.

El Enfoque Comunicativo, respaldado en el campo de la enseñanza de idiomas de manera amplia y profunda (Richards y Rodgers, 2001), hace "hincapié en la importancia de comprender el uso del lenguaje en una situación determinada y con unos objetivos específicos" (Talaván, 2010, p. 212). En este ámbito, y según la misma autora, las tareas de evaluación tienen que contemplar todas las formas y escenarios comunicativos que existen, y conseguir que estas emulen contextos auténticos e impredecibles. Los entornos tecnológicos promueven nuevos escenarios de enseñanza-aprendizaje que ayudan de manera natural a la transición desde enfoques más tradicionales al Enfoque Comunicativo.

\section{Cambio de rol del alumno y del profesorado}

El formato masivo posee una característica disruptiva en relación con otras formas de enseñanza online: el público masivo (Castrillo, 2014). Esta propiedad replantea el papel que han de adquirir los docentes donde la comunicación continua entre profesor-alumno no es posible. Las funciones del profesor que pasan a tener más peso son el diseño, la planificación del curso y sus contenidos, la facilitación, la curación, y la investigación. Se parte del hecho de que capturar y analizar digitalmente los datos tiene gran potencial para entender y mejorar la enseñanza y el aprendizaje y permite mejorar el entendimiento acerca de los procesos de enseñanza no solo de manera cualitativa, sino reforzando la perspectiva mediante la combinación con datos cuantitativos (Veletsianos et al., 2015). La triangulación de datos por diferentes métodos resulta la más propicia para comprender de manera más extensa los comportamientos del participante MOOC, y por qué participan de la manera que lo hacen.

\section{Perfil del participante MOOC: perfiles de aprendizaje y socioeconómicos}

Varios autores han categorizado los rasgos del participante en cursos masivos. Kizilcec et al. (2013) realizan una clasificación relacionada con la involucración con el curso y el grado de participación que resulta en cuatro perfiles: (1) completing, refiriéndose a aquellos que intentan realizar todas las evaluaciones independientemente de su resultado, (2) auditing, interactúan de manera principal con las 
lecciones en vídeo ofrecidas en el curso, pero no realizan evaluaciones, aunque suelen seguir el curso hasta su finalización, (3) disengaging, estudiantes que normalmente en el primer tercio del curso se desvinculan de él, aunque durante el tiempo que están son activos y (4) sampling, estudiantes que solo acceden casi de manera exclusiva a vídeos durante un tiempo limitado. Por otra parte, Anderson et al. (2014) clasifican a los participantes teniendo en cuenta para ello el acceso a los materiales y la entrega de tareas. Como resultado surgen: (1) viewers, los cuales de manera principal acceden al visionado de las lecciones, pero no entregan prácticamente ninguna actividad; (2) solvers, que sí entregan tareas pero por el contrario, no acceden prácticamente a la consulta de los materiales disponibles; (3) all-rounders, que son un término medio entre los dos anteriores, por lo que encuentran el balance entre consulta y entregas; (4) collectors, los cuales descargan el material disponible, pero no acceden al visionado de este, ni completan prácticamente tareas y (5) bystanders, que únicamente se registran en los cursos sin implicarse en ninguna de las dinámicas ofrecidas por ellos.

La consideración de factores directamente relacionados con el contexto personal del aprendiz como rasgos sociales, económicos, y culturales ya han sido contemplados por diversos autores (Bárcena et al., 2014; Beaven et al., 2014; ChacónBeltrán, 2014; Whitmer et al., 2017; Wolfenden et al., 2017; Yousef et al., 2014), que defienden este conocimiento adicional sobre el participante. La tendencia general de los MOOC indica que los participantes son de manera principal hombres de países desarrollados, de edad media, empleados, con estudios y cuya motivación principal para tomar los cursos es la promoción laboral (Christensen et al., 2013; Daniel et al., 2015).

\section{Herramientas y materiales usados en los LMOOC}

En el diseño instruccional de los LMOOC se suelen incluir materiales como lecciones en vídeo, artículos relacionados con temas sujetos a estudio, y herramientas para la interacción social que permiten al estudiante trabajar competencias lingüísticas de producción y recepción sin las restricciones de la educación tradicional (Sokolik, 2014). La interacción toma forma asíncrona para facilitar la viabilidad debido a la gran escala en la que se posicionan los cursos masivos a nivel de participación. Estos materiales y herramientas son de ayuda para el participante de MOOC de lenguas, ya que dan pie a la práctica de las diferentes competencias del lenguaje (Beaven et al., 2014).

De acuerdo con Glance et al. (2013), los LMOOC contienen elementos comunes entre sí: vídeos explicativos, corrección automática y entre compañeros, 
cuestionarios de retroalimentación, y herramientas para la comunicación y el trabajo colaborativo como los foros, blogs y wikis (Castrillo, 2014). El contenido audio visual al igual que el escrito puede estar clasificado por contenido para que el usuario pueda ajustarse, dentro del material ofrecido, a sus preferencias (Gafaro, 2020).

\section{Metodología}

El estudio ha seguido principios de investigación cualitativos. Primero se ha realizado un análisis bibliográfico donde el instrumento cualitativo es el análisis documental. Después se ha analizado el caso específico del LMOOC "How to succeed in the English B1 Level Exam" (1 ${ }^{a}$ edición). En este caso, la técnica cualitativa ha sido el cuestionario donde se recoge la satisfacción del participante. Los datos cuantitativos se han recogido a través de la grabación de la actividad de los participantes en la plataforma y un cuestionario que cubre aspectos demográficos, entre otros. La recopilación de muestras para el análisis bibliográfico se ha realizado en dos fases: revisión de monografías sobre LMOOC, y revisión de publicaciones periódicas en revistas científicas. Para ello, primero se realizó un barrido general a través de la herramienta de búsqueda "Google Académico", acotando la búsqueda con los términos: MOOC(s), "language” (s), “case study”, y “estudio de caso". En segundo lugar, se accedió a la herramienta de búsqueda proporcionada por la Biblioteca UNED, donde se realizó: (1) búsqueda simple: language(s) MOOC, y (2) búsqueda booleana: “language(s)" MOOC(s) AND “case study.” Por último, se accedió a la búsqueda básica y booleana en las bases de datos ERIC y WOS (Web of Science) siguiendo el mismo patrón.

Se han obtenido 17 estudios de caso que hacen mención específica a perfiles de comportamiento, perfiles socioeconómicos, y/o ambos. Los autores de dichos estudios son: Agonács et al. (2019); Bàrkàny y Melchor-Couto (2017); Beaven et al. (2014); Chacón-Beltrán (2014, 2017); Damevska (2015); Fuchs (2019); Gafaro (2020); Lee et al. (2018); Martín-Monje et al. (2014, 2018); Ozan et al. (2018); Pérez (2019); Rubio (2014); Santos et al. (2014); Sedano (2017); Ventura y MartínMonje (2016) y Zancanaro y Domingues (2018). Se detectó que la mayoría de los autores se centran en perfilar a los participantes en relación con su grado de involucración con el curso y el grado de participación que deriva en tasas de compleción. También se ha tenido en cuenta el uso de materiales y compleción de tareas evaluativas en las descripciones. 
Figura 1. Diagrama del proceso de recopilación de datos bibliográficos

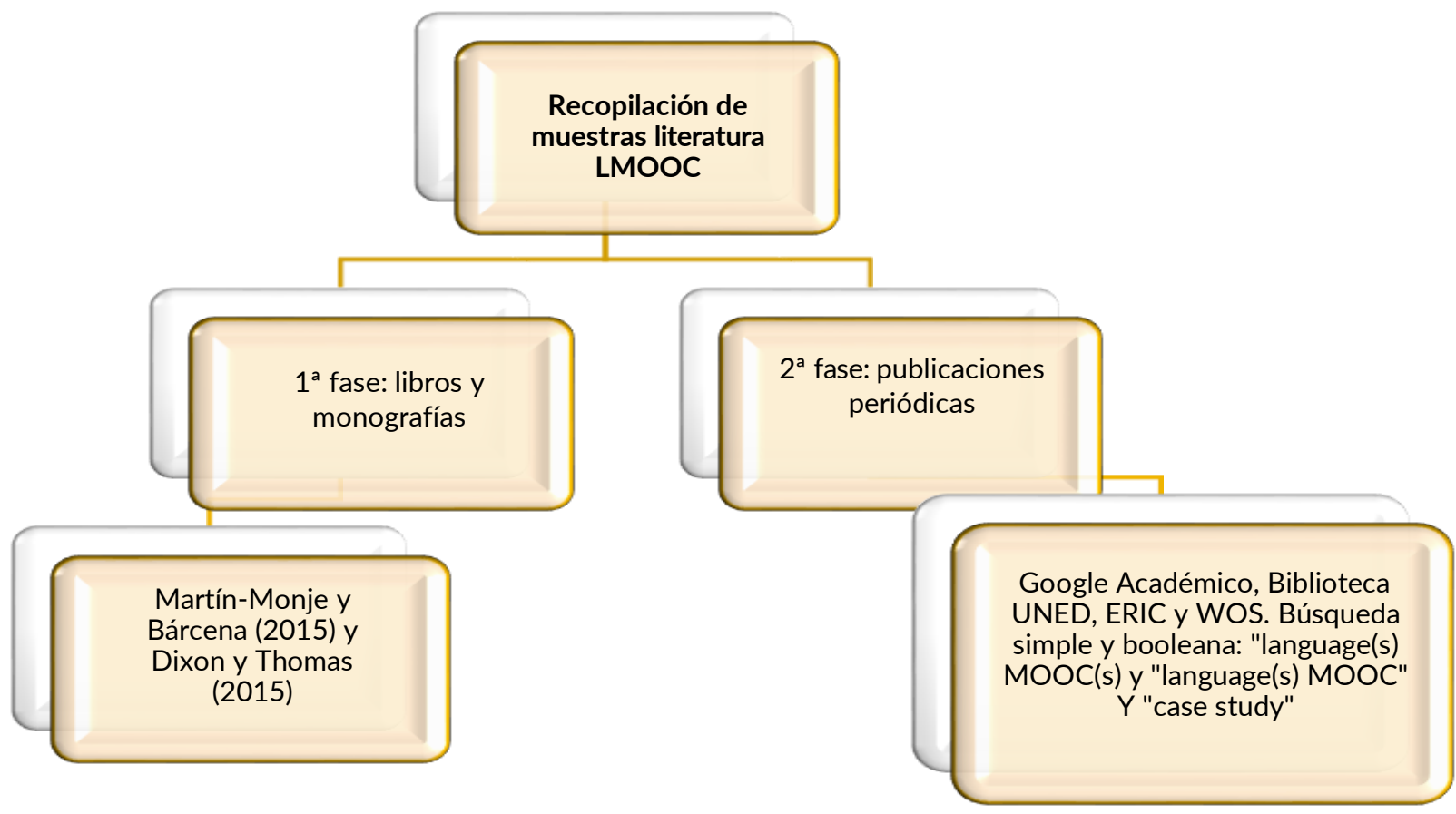

Para el análisis del estudio del caso específico "How to succeed in the English B1 Level Exam", se ha tenido acceso a los datos de la primera edición del LMOOC gracias a la cesión de estos por parte del proyecto $\mathrm{ECO}^{1}$ en el cual se desarrolla. Mediante el acceso a las respuestas de los cuestionarios y la monitorización del comportamiento en línea, se puede realizar un análisis de las rutas de aprendizaje que toman los participantes para así poder categorizarlos según las denominaciones de Kizilcec et al. (2013) y Anderson et al. (2014).

\section{DESCRIPCIÓN Y ANÁLISIS DE DATOS}

\section{PI1: Características socioeconómicas del participante en LMOOC}

Mediante el análisis bibliográfico se observa que los LMOOC son cursados en su mayoría por mujeres adultas jóvenes, empleadas, con estudios universitarios y provenientes de países desarrollados. En el Anexo I se reflejan los porcentajes más altos de cada rasgo encontrado en la literatura revisada.

\footnotetext{
${ }^{1}$ Proyecto ECO (E-Learning, Communication, and Open-data), [grant number 622127]:

https://cordis.europa.eu/project/id/621127/es
} 


\section{PI2: Perfiles relacionados con patrones de comportamiento del participante en LMOOC}

En respuesta a la PI2, donde se pretende determinar el perfil dominante en LMOOC en cuanto a patrones de comportamiento, se observa en la literatura (véase Anexo I) que los participantes no se involucran con el curso, lo que se traduce en bajas tasas de compleción y también abandono del curso de forma progresiva. Los estudiantes generalmente no entregan tareas evaluativas, aunque en el tiempo que permanecen en el curso son participantes activos que acceden a las herramientas de comunicación y al visionado de materiales. El perfil dominante, por lo tanto, es el viewer según Anderson et al. (2014). Se distingue otro perfil ligeramente usual: all-rounders, según los mismos autores.

\section{Perfiles socioeconómicos y de comportamiento dominante en el caso especifico}

La información facilitada por los participantes en el curso específico permite conocer su edad, sexo, nacionalidad, nivel de estudios y situación laboral (véase la tabla 1). Se define el perfil más relevante por el conjunto de características que han alcanzado un porcentaje más alto, resultando en: mujeres de mediana edad empleadas, con estudios universitarios, y provenientes de países desarrollados.

Tabla 1. Perfil demográfico

\begin{tabular}{ccccc}
\hline \multicolumn{4}{c}{ Perfil demográfico predominante de los participantes } \\
\hline $\begin{array}{c}\text { Nacionalidad } \\
\text { Española }\end{array}$ & Género & Edad & Nivel de estudios & Situación laboral \\
$(91,59 \%)$ & Femenino & 37,6 (media) & Universitarios & Empleados a tiempo \\
completo (43,48\%)
\end{tabular}

En cuanto a los perfiles de comportamiento, los descriptores utilizados para definirlos fueron: los recursos de aprendizaje utilizados por los participantes, la interacción y participación en línea y el progreso en el curso. La información sobre el comportamiento en línea fue procesada como parte de una investigación en el seno del Proyecto ECO con el programa computacional para el análisis estadístico SPSS.

El número de materiales o recursos disponibles es de 16 lecciones en vídeo, 12 documentos descargables y 2 textos en línea, siendo las lecciones en vídeo las más utilizadas, tal y como se puede observar en la tabla 2 donde se muestra la media de uso de los participantes. El número de entradas en los foros indica una participación baja y decreciente según avanza el curso (véase la tabla 3). La 
participación en la red social Facebook es de igual forma modesta, tan solo un 14\% de los estudiantes (747) que comienzan el curso la utilizan como herramienta adicional de comunicación.

El acceso a los materiales junto con la entrega de tareas y participación determina los perfiles de comportamiento (véase Tabla 4). Por último, se define el progreso en el curso mediante la compleción de este. En la tabla 5 se pueden observar las diferencias entre el número de estudiantes registrados, los que realmente empiezan el curso y los que logran terminarlo, lo que implica haber completado y entregado el conjunto de las actividades propuestas en cada módulo.

Tabla 2. Datos estadísticos descriptivos sobre la involucración con los objetos de aprendizaje mediante analíticas del aprendizaje (Proyecto ECO)

\begin{tabular}{lccc}
\hline \multicolumn{4}{l}{ Involucración con los objetos de aprendizaje: datos descriptivos } \\
\hline & Acceso a artículos & Acceso a vídeos & Acceso a libros \\
\hline $\mathrm{N}$ & 2600 & 4436 & 3087 \\
Media & 5.74 & 12.68 & 5.51 \\
Mediana & 3.00 & 7.00 & 4.00 \\
Moda & 1 & 2 & 1 \\
Desviación estándar & 7.558 & 16.792 & 5.519 \\
Asimetría & 3.371 & 6.918 & 2.533 \\
\hline
\end{tabular}

Tabla 3. Indicadores de participación en los foros

\begin{tabular}{ccccccccc}
\hline \multicolumn{8}{c}{ Participación en los foros } \\
\hline Denominación & Guías & General & Mód. 1 & Mód. 2 & Mód. 3 & Mód. 4 & Mód. 5 & Mód. 6 \\
Entradas & 250 & 54 & 505 & 100 & 87 & 80 & 44 & 41 \\
\hline
\end{tabular}

Tabla 4. Determinación de perfiles de comportamiento (Proyecto ECO)

\begin{tabular}{ccc}
\hline $\begin{array}{c}\text { Estilo de involucración } \\
\text { (Anderson et al., 2014) }\end{array}$ & Descripción & Frecuencia \\
\hline "Viewers" & Acceden a objetos de aprendizaje, pero no entregan tareas & 1614 \\
"All-rounders" & Acceso a mínimo 2 objetos de aprendizaje; mínimo una tarea & 1236 \\
"Solvers" & enviada y mínimo un comentario en el foro & 237 \\
"Bystanders" & Visualización de 1 vídeo sin entrega de tareas o participación en los foros & 211 \\
"Collectors" & Descarga materiales sin interacciones en línea & 49 \\
\hline
\end{tabular}


Tabla 5. Participación durante el curso (Proyecto ECO)

\begin{tabular}{ccc}
\hline \multicolumn{3}{c}{ Participación de los estudiantes } \\
\hline Registrados & Empiezan el curso & Completan el curso \\
8208 & $5359(65 \%)$ & $644(12 \%)$ \\
\hline
\end{tabular}

El bajo porcentaje de participantes que logran completar el curso pueden ser definidos como solvers según la taxonomía de Anderson et al. (2014). Su relación con los materiales es escasa, apenas acceden a ellos, pero sin embargo entregan una cantidad de tareas que les permite completar la formación.

El comportamiento de los participantes en relación con las herramientas de comunicación no es relevante y decrece de manera progresiva según avanza el curso. Este hecho, complementado con la poca cantidad de alumnos que lo finaliza, permite clasificar a los participantes según la taxonomía de Kizilcec et al. (2013) como disengaging o los que abandonan durante las fases iniciales. Tomando en cuenta esa misma parte de la población que no completa el curso $(88 \%)$ y su relación con los materiales y la entrega de tareas, se puede deducir que la mayoría de los participantes pueden ser definidos como viewers según Anderson et al. (2014). Acceden de manera habitual al visionado de materiales teniendo preferenica por las lecciones en vídeo, pero no alcanzan a entregar ninguna tarea evaluativa. Siguiendo con la misma taxonomía, los datos revelan que después de los viewers el comportamiento más habitual es el que define a los all-rounders.

La triangulación de datos cualitativos y cuantitativos (véase la tabla 6) provenientes del control sobre el acceso al curso, las analíticas del aprendizaje, y los cuestionarios, permiten validar los resultados. En primer lugar, los cuestionarios finales revelan una valoración positiva de los objetos de aprendizaje: artículos, libros y audio lecturas (33,2\%); superior a las tareas de evaluación colaborativas o en forma de quiz $(29,1 \%)$, teniendo en cuenta las valoraciones "muy buena" y "buena” mostradas en las figuras 2 y 3.

Tabla 6. Triangulación de datos caso específico: perfil predominante: "viewers"

\begin{tabular}{ccc}
\hline Cuestionario & Analíticas del aprendizaje & Control de acceso al curso \\
\hline $\begin{array}{c}\text { Máxima valoración: objetos de } \\
\text { aprendizaje (lecciones en vídeo) }\end{array}$ & $\begin{array}{c}\text { Máximo tiempo de consulta: } \\
\text { objetos de aprendizaje } \\
\text { (lecciones en vídeo) }\end{array}$ & $\begin{array}{c}\text { Máximo acceso a: } \\
\text { objetos de aprendizaje } \\
\text { (lecciones en vídeo) }\end{array}$ \\
\hline
\end{tabular}


Figura 2. Porcentajes de la valoración de las tareas de evaluación en el cuestionario final

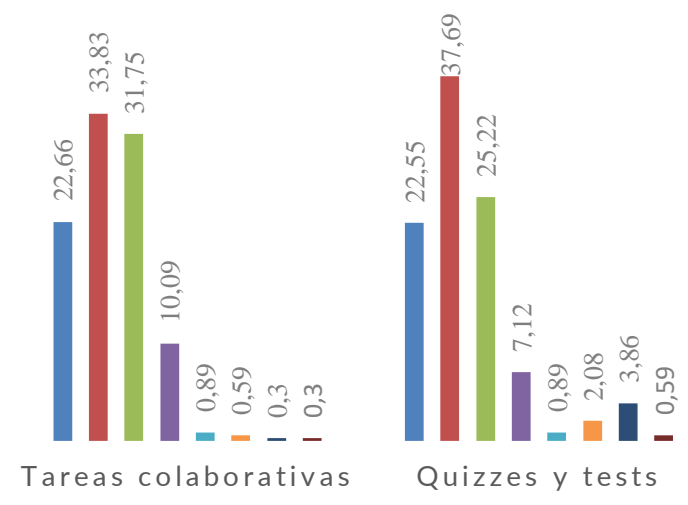

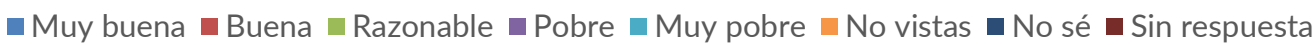

Figura 3. Porcentajes de la valoración de los objetos de aprendizaje en el cuestionario final

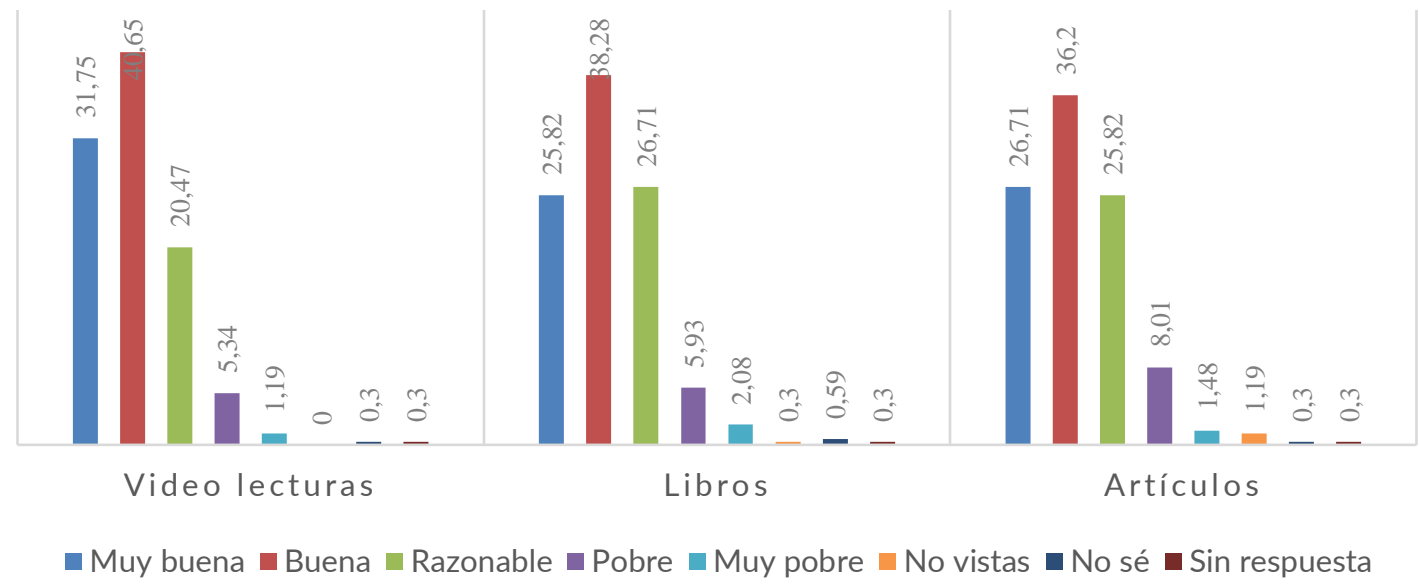

De igual forma, y según la estadística descriptiva, los participantes priorizan la consulta de recursos ante la entrega de tareas. Por otra parte, los datos muestran que teniendo en cuenta el tiempo que los participantes dedican al acceso a materiales didácticos, las lecciones en vídeo, categorizadas dentro de los objetos de aprendizaje, obtienen más tiempo de visualización, con tiempos superiores a los dedicados a la consulta y entrega de tareas, constatando el predominio del perfil viewers, según Anderson et al. (2014). Por último, el control sobre el acceso al curso muestra que los participantes que siguen activos eligen la visualización de las lecciones en vídeo como material didáctico de manera principal. Estos se involucran en mayor medida con este tipo de recursos durante el periodo que dura su formación, aunque los accesos decrecen a medida que avanza el curso. Los participantes que continúan hasta la compleción tienen obligación de entregar tareas con este fin. Con este dato se pasa a confirmar la asociación entre perfiles: all-rounders - completers, siendo los primeros los que encuentran balance entre consulta de materiales y entrega de 
tareas, lo que, de manera consecuente, les permite finalizar el curso tal y como está estructurado. El número de participantes que completan el curso es claramente minoritario, por lo que se confirma el perfil viewers gracias al control de acceso a las diferentes secciones del curso.

Las características socioeconómicas que definen a los participantes en el estudio bibliográfico y el estudio de caso son exactamente idénticas (véase la Tabla 1 y el Anexo I), obteniendo los porcentajes más altos en los mismos rasgos por lo que se responde a la PI4 confirmando la similitud de perfiles en el caso específico con la tendencia general.

En cuanto a los perfiles de comportamiento se revelan las similitudes entre el estudio de caso y la tendencia general en LMOOC. Se ha establecido que la combinación más recurrente dentro de la visión panorámica es la de viewers-disengaging, deduciendo una relación causal entre el abandono y la no entrega de tareas evaluativas. En el caso específico sucede lo mismo, la mayoría de las participantes coinciden con la descripción de la definición de Anderson et al., (2014): viewers, En este caso se deduce de igual manera que los viewers abandonan el curso de manera progresiva con mayor incidencia en los módulos finales, obteniendo la denominación disengaging que hace referencia a este hecho. De la misma forma, el segundo perfil más recurrente en ambos casos es el de all-rounders, poniendo de relieve que los estudiantes de MOOC de lenguas no tienen especial interés en completar el curso. En cuanto a la compleción del curso, relacionada con la entrega de tareas evaluativas, se deduce que no es una práctica habitual en ninguno de los casos.

\section{DISCUSIÓN Y CONCLUSIONES}

Existe una similitud en perfiles socioeconómicos de los participantes LMOOC con los MOOC en el resto de disciplinas, a excepción del género, que corroboran las barreras existentes para que este formato sea accesible a una extensión de la población más amplia y heterogénea. El matiz del género se asocia a la educación social y a una falta de referentes femeninos en estudios técnicos (Sáinz, 2017) lo que ha supuesto que la mujer se decante por el estudio de humanidades y ciencias sociales. También a una necesidad de comunicación asociada al género femenino (Pimentel, 2014) por la que el estudio de lenguas extranjeras les resulta más atractivo.

El hecho de que los LMOOC tengan características metodológicas distintivas no supone ningún cambio significativo en el patrón de perfiles explorados. Este hecho puede tener relación con la publicidad que se les da a los cursos masivos, no 
estando enfocada en los colectivos que más podrían beneficiarse de esta estructura (Bárcena et al., 2014). Los participantes son estudiantes que no dependen únicamente de esta modalidad instruccional para formarse, dejando fuera a colectivos menos favorecidos. Conscientes de la situación, diversos proyectos proponen los LMOOC como opción de integración para colectivos en riesgo de exclusión, haciendo que las plataformas se adapten a un público multicultural y multilingüe (Vázquez-Cano et al., 2016). También influye el uso del idioma inglés como "lingua franca", lo que supone un dilema en los LMOOC dedicados a la propia enseñanza del inglés siendo el objetivo y el medio a la misma vez (Martín-Monje, 2019).

El hecho de categorizar al participante como una entidad única (Knox, 2014) propicia la rigidez en estructuras didácticas, no dando pie a una adaptación más personalizada. Este hecho puede ser solventado con el uso de técnicas del Learning Analytics, que permitan rastrear preferencias y adecuarlas a las necesidades específicas de cada participante, teniendo la posibilidad de intervención para la propuesta de nuevas rutas antes de un posible abandono por no encontrar un camino adecuado a las necesidades particulares del usuario.

Es llamativo cómo las herramientas de comunicación no logran suscitar interés en una parte importante de los usuarios. Estas son esenciales en el aprendizaje de lenguas, ya que permiten desarrollar la competencia comunicativa e implícitamente promueven la práctica de competencias lingüísticas (Beaven et al., 2014; Sokolik, 2014). Una propuesta ante este hecho es dar peso a tareas relacionadas con la interacción entre los participantes que fomenten actos comunicativos, y sean más significativas para aprendices de lenguas extranjeras, en detrimento de tareas evaluativas tradicionales como test autocorrectivos y similares.

La apertura hacia caminos de aprendizaje cada vez más personalizados y participativos permite el replanteamiento de la definición de éxito en los LMOOC y MOOC en general, que históricamente ha sido relacionado con la compleción o el abandono del curso. La combinación de perfiles hallada más habitual en los participantes de LMOOC, de manera general y en el caso específico, es la de disengaging-viewers. Esto puede significar que los estudiantes LMOOC y MOOC en general no tienen un interés o motivación especial en completar la formación cumpliendo los requisitos definidos para este fin, que va ligado a la entrega de tareas evaluativas y a la participación. Godwin-Jones (2014) y Beaven et al., (2014) entienden que este comportamiento es comprensible por la naturaleza gratuita y abierta de los cursos y la visión que tienen los participantes de tomar la experiencia MOOC como la asistencia a una conferencia donde no es necesaria la asistencia 
continua, y no como un curso al uso. Sin embargo, los participantes con este perfil de comportamiento son activos y seleccionan los materiales a consultar antes del cese de su actividad en el curso. Un amplio número de usuarios decide no realizar las tareas, aunque aprovechan el curso de otras maneras como con la descarga y visualización de materiales y acceso a determinados recursos desechando trabajar el resto por no ser de su interés particular. Puede entenderse que el tiempo que permanecen activos es provechoso para ellos y los motivos de abandono tengan que ver con el hecho de haber cumplido sus objetivos personales y no la totalidad de objetivos propuesta por el curso. Este comportamiento denota autonomía por parte del participante, ya que los recursos que se utilizan son los que consideran útiles para complementar su formación.

Por lo tanto, sería conveniente prestar atención a aspectos como la utilidad del contenido de manera individual independientemente de la compleción, y medir la satisfacción de los perfiles mayoritarios. Esto permitiría deducir si los cursos son provechosos para los perfiles predominantes y replantear el sentido del éxito asociado a la compleción mediante entrega de tareas. La elaboración de cuestionarios iniciales puede cubrir esa necesidad y dar voz a los usuarios con necesidades específicas de aprendizaje. Los cuestionarios han de dirigirse a la averiguación del interés individual en ciertos contenidos, y de las expectativas del participante, que son las que habrían de marcar su éxito o fracaso.

Las taxonomías que hasta ahora han dado nombre al tipo de perfil de estudiante LMOOC y relacionadas con el comportamiento del participante podrían ser complementadas con esta información adicional y específica sobre sus intenciones para con el curso. Las propuestas didácticas innovadoras, como son el caso que nos ocupa, no pueden medir el éxito de la experiencia con métodos tradicionales, por lo que es interesante buscar alternativas que reflejen las distintas realidades que se dan en los LMOOC.

\section{LIMITACIONES DEL ESTUDIO Y FUTURAS VÍAS DE INVESTIGACIÓN}

Los resultados presentados en la primera parte del estudio están recogidos mediante una búsqueda exhaustiva de la literatura existente en el campo del estudio de lenguas a través del formato MOOC. Aunque la tendencia general es clara tras los resultados provenientes de las muestras recopiladas, conviene remarcar que la generalización que se presume en el estudio no representa a la totalidad de LMOOC existentes. Por otra parte, el estudio ha clasificado a los participantes según patrones de comportamiento. La diversidad en la presentación de estos datos en la 
literatura consultada ha obligado a buscar el mayor número de similitudes en los datos obtenidos con las taxonomías de Kizilcec et al. (2013) y Anderson et al., (2014) que sirven de complemento para explicar los datos hallados. Esto supone que los perfiles presentados en el estudio no representen de manera completa los rasgos definitorios de las clasificaciones de los autores. Por último, remarcar que tanto en los casos estudiados en la revisión bibliográfica y en el caso específico "How to succeed in the English B1 Level Exam", los datos socioeconómicos no representan al total de los participantes.

Tras el estudio de los datos presentados, se abre un gran abanico de oportunidades a aplicar en la práctica. Estas abarcan cuestiones desde el propio diseño relacionado con: la metodología, contenidos, seguimiento del participante y aprovechamiento; pasando por la manera en que se ofrecen los LMOOC y el público objetivado, hasta la aplicación de propuestas que logren romper las barreras existentes. Estas posibilidades reflejan una realidad en la que el formato LMOOC es susceptible y tiene el potencial de integrar elementos que enfoquen la enseñanza en un público más amplio, autónomo e interesado en la posibilidad de acceder a formación a lo largo de la vida siguiendo sus propios objetivos de aprendizaje. Lo cierto es que la propia naturaleza abierta, flexible y modular de los LMOOC permite aprovechar al máximo la experimentación basada en combinaciones entre los elementos que conforman un curso para lograr encontrar modelos adaptativos que puedan dar cobertura formativa a más usuarios. Estos modelos tienen que respetar las necesidades de los estudiantes de lengua extranjera donde la práctica real del idioma a estudio por medio de las interacciones se sabe primordial.

\section{AgradeCimientos}

Agradezco al proyecto europeo ECO (E-Learning, Communication, and Opendata), [grant number 622127] la inestimable ayuda y la generosidad en la cesión de los datos resultantes de la primera edición del LMOOC "How to succeed in the English B1-Level exam", muy en especial a mi directora de tesis Elena Martín Monje. 


\section{ANEXO I: DESGLOSE DE DATOS DE LAS PUBLICACIONES CONSULTADAS}

\begin{tabular}{|c|c|c|c|}
\hline Referencia (A-Z) & Título LMOOC & Mención a perfiles de comportamiento & $\begin{array}{l}\text { Mención a perfiles } \\
\text { socioeconómicos }\end{array}$ \\
\hline $\begin{array}{l}\text { Agonács, N., y } \\
\text { Matos, J. F. (2019) }\end{array}$ & $\begin{array}{l}\text { "AP Italian Language } \\
\text { and Culture" }\end{array}$ & $\begin{array}{l}\text {-Disengaging: } 11 \text { de } 740 \text { participantes terminan } \\
\text { el curso. } \\
\text {-Viewers: los participantes acceden a materiales. }\end{array}$ & $\begin{array}{l}\text {-Muestra no representativa: } \\
\text { únicamente } 11 \text { participantes aportan } \\
\text { estos datos. }\end{array}$ \\
\hline $\begin{array}{l}\text { Bàrkànyi, Z., y } \\
\text { Melchor-Couto, S. } \\
\text { (2017) }\end{array}$ & "Spanish for beginners" & $\begin{array}{l}\text {-Solvers / All- rounders: Se indica que el } 50 \% \\
\text { realiza las tareas, sin hacer mención directa al } \\
\text { acceso a materiales. }\end{array}$ & No aporta datos \\
\hline $\begin{array}{l}\text { Beaven, T., } \\
\text { Codreanu, T. y } \\
\text { Creuzé, A. (2014) }\end{array}$ & "Travailler en français" & $\begin{array}{l}\text {-Disengaging } \\
\text {-Viewers: la mayoría accede a las lecciones en } \\
\text { video, pero un bajo porcentaje presenta tareas } \\
\text { evaluativas. }\end{array}$ & $\begin{array}{l}\text {-Situación laboral: empleados (46,3\%) } \\
\text {-Origen: España (36\%), seguido de } \\
\text { Francia e Italia }\end{array}$ \\
\hline $\begin{array}{l}\text { Chacón-Beltrán, R. } \\
\text { (2017) }\end{array}$ & $\begin{array}{l}\text { "Beginners' English } \\
\text { Course for Spanish } \\
\text { Speakers" }\end{array}$ & $\begin{array}{l}\text {-Viewers: solo el } 24 \% \text { de los participantes activos } \\
\text { en el curso entregan tareas. }\end{array}$ & $\begin{array}{l}\text {-Edad: } 25-45(63,05 \%) \\
\text {-Sexo: mujer (68\%) } \\
\text {-Estudios: universitarios (55\%) } \\
\text {-Situación laboral: empleados (38,17\%) } \\
\text {-Origen: España (80.09\%) }\end{array}$ \\
\hline $\begin{array}{l}\text { Chacón-Beltrán, R. } \\
\text { (2014) }\end{array}$ & $\begin{array}{l}\text { "Empieza con } \\
\text { el inglés/English } \\
\text { Beginners" }\end{array}$ & $\begin{array}{l}\text {-Completing: el } 55 \% \text { entrega las tareas } \\
\text { evaluativas. } \\
\text {-All-rounders: el } 50 \% \text { entrega tareas y accede a } \\
\text { los materiales. }\end{array}$ & $\begin{array}{l}\text {-Edad: } 30-39(32 \%) \\
\text {-Sexo: mujer (68\%) } \\
\text {-Estudios: universitarios (55\%) } \\
\text {-Origen: España (97\%) }\end{array}$ \\
\hline Damevska, L. (2015). & "Tandem MOOC" & -Disengaging & $\begin{array}{l}\text {-Edad: } 26-35(32,1 \%) \\
\text {-Sexo: mujer }(62 \%) \\
\text {-Origen: España }(43,9)\end{array}$ \\
\hline Fuchs, C. (2019) & $\begin{array}{l}\text { "Understanding } \\
\text { Language: Learning and } \\
\text { Teaching" }\end{array}$ & $\begin{array}{l}\text {-Auditing } \\
\text {-All-rounders: Interactúan con los materiales y } \\
\text { terminan el curso, aunque entregan pocas tareas } \\
\text { evaluativas. }\end{array}$ & No aporta datos \\
\hline
\end{tabular}

Gafaro, B. C. (2020) "English for Academic
Purposes"
-Completing: MOOC integrado en educación formal, el total de los participantes lo completan.
-Estudios: universitarios

\begin{tabular}{|c|c|c|c|}
\hline $\begin{array}{l}\text { Lee, S., Kadel, R. S., } \\
\text { Madden, A. y Gazi, } \\
\text { Y. (2018) }\end{array}$ & $\begin{array}{l}\text { "Speak English } \\
\text { Professionally: In } \\
\text { Person, Online y On the } \\
\text { Phone" }\end{array}$ & $\begin{array}{l}\text {-Disengaging: solo el } 3,9 \% \text { de los participantes } \\
\text { termina el curso. } \\
\text {-Viewers: presentan pocas evaluaciones y } \\
\text { acceden al material audiovisual. }\end{array}$ & $\begin{array}{l}\text {-Edad (media): } 33 \\
\text {-Sexo: mujer (52,7\%) } \\
\text {-Estudios: universitarios (67\%) } \\
\text {-Situación laboral: empleados }(45,2 \%)\end{array}$ \\
\hline $\begin{array}{l}\text { Martín-Monje, E., } \\
\text { Bárcena, E., y } \\
\text { Read,T. (2014). }\end{array}$ & $\begin{array}{l}1^{\text {a }} \text { edición: "Inglés } \\
\text { profesional" }\end{array}$ & $\begin{array}{l}\text {-Disengaging } \\
\text {-Viewers: La participación decrece según avanza } \\
\text { el curso. }\end{array}$ & $\begin{array}{l}\text {-Edad: } 25-35(46,11 \%) \\
\text {-Estudios: universitarios (55,33\%) } \\
\text {-Origen: España (60-80\%) }\end{array}$ \\
\hline $\begin{array}{l}\text { Martín-Monje, E., } \\
\text { Castrillo, M. D. y } \\
\text { Mañana-Rodríguez, } \\
\text { J. (2018). }\end{array}$ & $\begin{array}{l}\text { "How to succeed in the } \\
\text { B1-level exam" }\end{array}$ & $\begin{array}{l}\text {-Viewers: mención específica a esta } \\
\text { denominación del perfil. }\end{array}$ & No aporta datos \\
\hline
\end{tabular}




\begin{tabular}{|c|c|c|c|}
\hline $\begin{array}{l}\text { Ozan, O., Ozarslan, } \\
\text { Y. y Yıldız, H. (2018) }\end{array}$ & $\begin{array}{l}\text { "Türkçe Öğreniyorum" } \\
\text { (Learn Turkish) }\end{array}$ & No aporta datos & $\begin{array}{l}\text {-Edad: } 18-24(40,87 \%) \\
\text {-Sexo: hombre }(65,8 \%) \\
\text {-Estudios: universitarios (44\%) } \\
\text {-Situación laboral: empleados (44\%) } \\
\text {-Origen: Turquía }(66,49 \%)\end{array}$ \\
\hline Pérez, J. J. (2019) & $\begin{array}{l}3^{\text {a }} \text { edición: "Inglés } \\
\text { profesional/ } \\
\text { Professional English" }\end{array}$ & $\begin{array}{l}\text {-Desengaging: Se accede a los materiales en los } \\
\text { módulos iniciales, luego decrece el acceso a } \\
\text { estos. }\end{array}$ & No aporta datos \\
\hline Rubio, F. (2014). & $\begin{array}{l}\text { "Improving your } \\
\text { Spanish pronunciation" }\end{array}$ & No aporta datos & $\begin{array}{l}\text {-Estudios: universitarios ( } 45 \% \\
\text { aproximadamente) }\end{array}$ \\
\hline $\begin{array}{l}\text { Santos, J. L., Klerkx, } \\
\text { J., Duval, E., Gago, } \\
\text { D., y Rodríguez, L. } \\
\text { (2014) }\end{array}$ & $\begin{array}{l}\text { "Fundamentals } \\
\text { of German for Spanish } \\
\text { speakers" }\end{array}$ & $\begin{array}{l}\text {-Disengaging: el } 50 \% \text { de los participantes deja el } \\
\text { curso tras los dos primeros módulos, con } \\
\text { posterior aumento progresivo en la tasa de } \\
\text { abandono. }\end{array}$ & No aporta datos \\
\hline Sedano, B. (2017). & "Spanish for travelling" & $\begin{array}{l}\text {-Disengaging: del } 70 \% \text { de los participantes que } \\
\text { empieza el curso solo un } 17 \% \text { lo completa con la } \\
\text { entrega de evaluaciones. No hace mención a } \\
\text { materiales pero por el contexto se puede deducir } \\
\text { que el } 59 \% \text { interactúa con los materiales aunque } \\
\text { no presenten evaluación. } \\
\text {-Viewers }\end{array}$ & $\begin{array}{l}\text {-Edad: } 20-40(52,8 \%) \\
\text {-Sexo: mujer (77,8\%) } \\
\text {-Estudios: universitarios (73\%) } \\
\text {-Situación laboral: empleados (75,6\%) } \\
\text {-Origen: Reino Unido y Estados Unidos } \\
\text { mayoritariamente. }\end{array}$ \\
\hline $\begin{array}{l}\text { Ventura, P., y } \\
\text { Martín-Monje, E. } \\
\text { (2016). }\end{array}$ & $\begin{array}{l}2^{a} \text { edición: } \\
\text { "Professional English" }\end{array}$ & $\begin{array}{l}\text {-Completing: } 58 \% \text { de los participantes completa } \\
\text { el curso }\end{array}$ & No aporta datos \\
\hline $\begin{array}{l}\text { Zancanaro, A. y } \\
\text { Domingues, M. J. de } \\
\text { Souza (2018) }\end{array}$ & $\begin{array}{l}\text { "Portuguese course for } \\
\text { foreigns" }\end{array}$ & $\begin{array}{l}\text {-Disengaging: el } 22 \% \text { de los participantes } \\
\text { completa el curso }\end{array}$ & $\begin{array}{l}\text {-Sexo: mujer }(54,8 \%) \\
\text {-Estudios: universitarios (46,76\%) } \\
\text {-Origen: España }(29,63 \%)\end{array}$ \\
\hline
\end{tabular}

\section{REFERENCIAS}

Agonács, N., \& Matos, J.F. (2019). Understanding language MOOC learners: The issue of capability development. International Journal of Emerging Technologies in Learning, 14(11), 123-137. https://doi.org/10.3991/IJET.V14I11.10205

Anderson, A., Huttenlocher, D., Kleinberg, J. \& Leskovec, J. (2014). Engaging with massive online courses. In Proceedings of the $23^{\text {rd }}$ international conference on World Wide Web (WWW 14). Nueva York. Association for Computing Machinery, 687-698. https://doi.org/10.1145/2566486.2568042

Atenas, J. (2015). Modelo de democratización de los contenidos albergados en los MOOC. RUSC. Universities and Knowledge Society Journal, 12(1). 3-14. http://dx.doi.org/10.7238/rusc.v12i1.2031

Bárcena, E., Read, T., Martin-Monje, E., \& Castrillo, M. D. (2014). Analysing student participation in Foreign Language MOOCs: a case study. EMOOCs 2014: European MOOCs Stakeholders Summit, 11-17.

Bárkányi, Z., \& Melchor-couto, S. (2017). Foreign language anxiety on a massive open online course. In CALL in a climate of change: Adapting to turbulent global conditions-Short 
papers from the EUROCALL conference, 24-29. https://doi.org/10.14705/rpnet.2017.eurocall2017.683

Beaven, T., Codreanu, T. \& Creuzé, A. (2014). Motivation in a language MOOC: issues for course designers. In: Elena Martín-Monje \& Elena Bárcena (Ed.), Language MOOCs: Providing Learning, Transcending Boundaries (pp. 48-66). De Gruyter Open.

Castrillo, M.D. (2014). Language Teaching in MOOCs: the Integral Role of the Instructor. In Elena Martín-Monje and Elena Bárcena (Ed.), Language MOOCs (pp. 67-90). De Gruyter Open Poland. https://doi.org/10.2478/9783110420067.5

Castrillo, M.D.; Martín-Monje, E. \& Vázquez-Cano, E. (2018). Guía práctica para el diseño y tutorización de MOOC. Telefónica Educación Digital. https://aleesp.hypotheses.org/category/articulos-resenas-publicaciones

Chacón-Beltrán, R. (2014). Massive online open courses and language learning: the case for a beginners' English course. Procedia-Social and Behavioral Sciences, 141, 242-246. https://doi.org/10.1016/j.sbspro.2014.05.042

Chacón-Beltrán, R. (2017). The role of MOOCs in the learning of languages: Lessons from a Beginners' English Course. Porta Linguarum: revista internacional de didáctica de las lenguas extranjeras, 28, 23-35. https://doi.org/10.30827/Digibug.54001

Christensen, G., Steinmetz, A., Alcorn, B., Bennett, A., Woods, D., \& Emanuel, E. (2013). The MOOC Phenomenon: Who Takes Massive Open Online Courses and Why? SSRN Electronic Journal. http://dx.doi.org/10.2139/ssrn.2350964

Conole, G. (2016). Los MOOC como tecnologías disruptivas: estrategias para mejorar la experiencia de aprendizaje y la calidad de los MOOC. Revista de Educación a Distancia, 50. https://revistas.um.es/red/article/view/270791

Cormier, D. \& Siemens, G. (2010). The Open Course: Through the Open Door: Open Courses as Research, Learning, and Engagement. Educause Review. 45(4), 30-32. https://eric.ed.gov/?id=EJ951238

Damevska, L. (2015). Students' perceptions of learning and networking in Tandem MOOC: a case study. Tesis de doctorado inédita, Universitat Rovira i Virgili.

Daniel, J.; Vázquez-Cano, E. \& Gisbert, M. (2015). El futuro de los MOOC: ‘aprendizaje adaptativo o modelo de negocio? RUSC. Universities and Knowledge Society Journal, 12, 64-73. http://dx.doi.org/10.7238/rusc.v12i1.2475

Dixon, E., \& Thomas, M. (2015). Researching language learner interactions online: From social media to MOOCs, 13. CALICO.

Fuchs, C. (2019). The structural and dialogic aspects of language massive open online courses (LMOOCs): A case study. In C. Wang \& L. Winstead (Eds.), Computer-Assisted language learning: concepts, methodologies, tools, and applications (pp. 1540-1562). https://doi.org/10.4018/978-1-5225-0177-0.ch002

Gafaro, B. C. (2020). MOOCs in the Language Classroom: Using MOOCs as Complementary Materials to Support Self-Regulated Language Learning. In A. Andujar (Eds.), Recent Tools for Computer- and Mobile-Assisted Foreign Language Learning (pp. 194-211). IGI Global. https://doi.org/10.4018/978-1-7998-1097-1.ch009

Glance, D. G., Forsey, M., \& Riley, M. (2013). The pedagogical foundations of massive open online courses. First Monday, 18(5). https://doi.org/10.5210/fm.v18i5.4350

Godwin-Jones, R. (2014). Global reach and local practice: The promise of MOOCS. Language Learning \& Technology, 18(3), 5-15. http://dx.doi.org/10125/44377

Kizilcec, R., Piech, C., \& Schneider, E. (2013). Deconstructing disengagement: analyzing learner subpopulations in massive open online courses. In Proceedings of the third international conference on learning analytics and knowledge (pp. 170-179). https://doi.org/10.1145/2460296.2460330 
Knox, J. (2014) Digital culture clash: "massive" education in the E-learning and Digital Cultures MOOC. Distance Education, 35(2), 164-177.

https://doi.org/10.1080/01587919.2014.917704

Lee, S., Kadel, R.S., Madden, A., \& Yakut, G.A.Z.I. (2018). How can learner analytics data inform language MOOC design? Boğaziçi Üniversitesi Eğitim Dergisi, 35(1), 19-29. Retrieved from https://dergipark.org.tr/tr/download/article-file/627728

Martín-Monje, E. (2019). La creación de recursos educativos abiertos y MOOC en lenguas extranjeras. En J. Martin, M. I. Jiménez, A. Fernández \& C. Duée (Coord.) Tecnologías Integradas en la Didáctica de Lenguas Extranjeras (TIDLE) (pp. 45-58). Ministerio de Educación y Formación Profesional/Universidad de Castilla-La Mancha.

Martín-Monje, E., Bárcena, E., \& Read, T. (2014). Peer-to-peer interaction and linguistic feedback in foreign language MOOCS. Profesorado, Revista de Currículum y Formación del Profesorado, 18(1), 167-183. http://www.ugr.es/ recfpro/rev181ART10.pdf

Martín-Monje, E., Castrillo, M. D., \& Mañana-Rodríguez, J. (2018). Understanding online interaction in language MOOCs through learning analytics. Computer Assisted Language Learning, 31(3), 251-272. https://doi.org/10.1080/09588221.2017.1378237

Ozan, O., Ozarslan, Y. \& Yıldız, H. (2018). LMOOC Design and Development Process for an Agglutinative Language: “Türkçe Öğreniyorum” Case. Turkish Online Journal of Educational Technology, 2. 140-149. https://members.aect.org/pdf/Proceedings/proceedings $18 / 2018 \mathrm{i} / 18$ 15.pdf

Pérez, J. J. (2019). Patrones temporales de participación en MOOC. Estudio de un MOOC de lenguas. RIED. Revista Iberoamericana de Educación a Distancia, 22(2), 287-303. https://doi.org/10.5944/ried.22.2.23109

Pimentel, A. (2014, 8 de marzo). Las mujeres lideran el ranking de los idiomas con una mayor capacidad lingüística. Revista digital INESEM. https://revistadigital.inesem.es/idiomas/lasmujeres-lideran-el-ranking-de-los-idiomas/

Richards, J. C. \& Rodgers, T. S. (2001). Approaches and methods in language teaching. Cambridge University Press.

Ross, J., Sinclair, C., Knox, J., Bayne, S., \& Macleod, H. (2014). Teacher experiences and academic identity: The missing components of MOOC pedagogy. MERLOT Journal of Online Learning and Teaching, 10(1), 57-69. https://jolt.merlot.org/Vol10_No1.htm

Rubio, F. (2014). Boundless Education: The case of a Spanish MOOC. FLTMAG. https://fltmag.com/the-case-of-a-spanish-mood

Sáinz, M. (2017). Se buscan ingenieras, físicas y tecnólogas. ¿Por qué no hay más mujeres STEM? Fundación Telefónica. Recuperado de https:/www.fundaciontelefonica.com/cultura-digital/publicaciones/590/

Santos, J.L., Klerkx, J., Duval, E., Gago, D., \& Rodríguez, L. (2014). Success, activity, and dropouts in MOOCs. An exploratory study on the UNED COMA courses. ACM International Conference Proceeding Series, 98-102. https://doi.org/10.1145/2567574.2567627

Sedano, B. (2017). La atención a las necesidades y demandas específicas del alumnado en un mundo globalizado: el caso de un MOOC de español para viajar. RIED. Revista Iberoamericana de Educación a Distancia, 20(1), 161-182.

https://doi.org/10.5944/ried.20.1.16692

Sokolik, M. (2014). What constitutes an effective Language MOOC. En E. Martín- Monje y E. Bárcena, (Eds.), Language MOOCs: Providing Learning,Transcending Boundaries (pp. 16-32). De Gruyter Open. https://doi.org/10.2478/9783110420067.2

Talaván, N. (2010). Claves para comprender la destreza de la comprensión oral en lengua extranjera. Epos: Revista de Filología, 26, 195-216.

https://doi.org/10.5944/epos.26.2010.10643 
Türkay, S.; Eidelman, H; Rosen, Y.; Seaton, D.; López, G. \& Whitehill, J. (2017). Getting to know English language learners in MOOCs: their motivations, behaviours, and outcomes. In Proceedings of the Fourth (2017) ACM Conference on Learning and Scale (L@S '17) (pp. 209-212). https://doi.org/10.1145/3051457.3053987

Vázquez-Cano, E., López-Meneses, E., \& Barroso-Osuna, J. (2016). El futuro de los MOOC. Retos de la formación on-line, masiva y abierta. Educatio Siglo XXI, 34(1), 245- 247.

Veletsianos, G.; Collier, A. \& Schneider, E. (2015). Digging Deeper into Learners' Experiences in MOOCs: Participation in social networks outside of MOOCs, Notetaking, and contexts surrounding content consumption. British Journal of Educational Technology, 46, 570587. https://doi.org/10.1111/bjet.12297

Ventura, P., \& Martín-Monje, E. (2016). Learning specialized vocabulary through Facebook in a massive open online course. In A. Pareja-Lora, C. Calle-Martínez, \& P. Rodríguez-Arancón (Eds.), New perspectives on teaching and working with languages in the digital era (pp. 117-128). https://doi.org/10.14705/rpnet.2016.tislid2014.427

Whitmer, J., Schiorring, E., \& James, P. (2014). Patterns of persistence: what engages students in a remedial English writing MOOC? In Proceedings of the Fourth International Conference on Learning Analytics and Knowledge (pp. 279-28). https://doi.org/10.1145/2567574.2567601

Wolfenden, F., Cross, S., \& Henry, F. (2017). MOOC adaptation and translation to improve equity in participation. Journal of Learning for Development-JL4D, 4(2). https://j14d.org/index.php/ej14d/article/view/209/243

Yousef, A., Chatti, M., Schroeder, U. \& Wosnitza, Y. (2014). What Drives a Successful MOOC? An Empirical Examination of Criteria to Assure Design Quality of MOOCs. IEEE 14th International Conference on Advanced Learning Technologies, Athens (pp. 44-48). https://doi.org/10.1109/ICALT.2014.23

Zancanaro, A., \& Domingues, M.J.C. de S. (2018). Massive Open Online Courses (MOOC) for teaching Portuguese for foreigners: A case study. Turkish Online Journal of Distance Education, 19(2), 4-20. https://doi.org/10.17718/tojde.415602

\section{PAZ DíEZ-ARCóN}

Investigadora en formación. Líneas de trabajo: aprendizaje de lenguas asistidas por ordenador, enseñanza en abierto y metodologías de aprendizaje para la innovación educativa. Proyectos: Erasmus +, Innovación Docente e I+D+I. He sido profesora asociada en la UNED y he participado en congresos nacionales e internacionales. Revisora de “Lengua y Migración”.

pdiez@flog.uned.es https://orcid.org/0000-0002-4095-7881

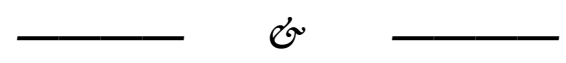

Díez-Arcón, P. (2021). Perfiles del participante en LMOOC: análisis bibliográfico y estudio de caso. Bellaterra Journal of Teaching \& Learning Language \& Literature, 14(3), e979. https://doi.org/10.5565/rev/jtl3.979 
Rebut / Recibido / Received / Reçu: 11-16-2020

Acceptat / Aceptado / Accepted / Accepté: 29-12-2021

https://revistes.uab.cat/jtl3/ 